Int. J. Dev. Biol. 52: 1099-1103 (2008)

doi: $10.1387 / \mathrm{ijdb} .082646 \mathrm{sd}$

\title{
Dicer inactivation causes heterochronic retinogenesis in Xenopus laevis
}

\author{
SARAH DECEMBRINI, MASSIMILIANO ANDREAZZOLI, GIUSEPPINA BARSACCHI and FEDERICO CREMISI* \\ Department of Biology, University of Pisa, Italy
}

\begin{abstract}
Maturation of miRNAs by dicer is required in vertebrates for normal neural development. Here we report that dicer inactivation in Xenopus affects cell cycle progression, survival and timing of the generation of retinal cells, resulting in small retinas with lamination defects. In particular, dicer inactivation delays the exit from the cell cycle and the translation of key genes of late neurogenesis, highlighting a crucial role of miRNAs in retinal development.
\end{abstract}

KEY WORDS: Dicer, miRNA, timing, neurogenesis, retina

\section{Introduction}

MicroRNAs play a key role in timing the development of the worm Caenorhabditis elegans (Ambros, 2000). In vertebrates, they are required for normal development (Bernstein et al., 2003; Giraldez et al., 2005; Harfe et al., 2005; Wienholds et al.,2005), but their precise role is still not fully understood. In zebrafish, inactivation of dicer, an enzyme that is required for miRNA maturation (Bartel, 2004), causes size reduction of nervous system structures, including the neural retina (Giraldez et al., 2005). However, little is known on the role of miRNAs in the control of cell cycle and cell fate determination of neural progenitor cells.

The different retinal cell types are generated from a common progenitor cell and exit from the cell cycle following an evolutionarily conserved timing schedule (ganglion cells, horizontal cells, cones, amacrine cells, rods, bipolar cells, Muller glia; Livesey et al., 2001). bHLH and homeobox genes (Hatakeyama et al., 2001) work together with cell cycle progression (Ohnuma et al., 2002; Cremisi et al., 2003) to establish the different retinal cell types. Recently, we described a cell-cycle-dependent clock, which sets the time of translation of key homeobox genes supporting the generation of photoreceptors (Xotx5b) and bipolar cells (Xotx2) in Xenopus developing retina (Decembrini et al., 2006). Since our observations indicate a key role of translational control in retinogenesis, we investigated the effects of miRNAs inactivation in Xenopus embryonic retina obtained by dicer knockdown.

\section{Results}

A tBlastx search identified a Xenopus clone (GenBank accession no. BP673528) displaying a highly significant similarity to all the described Dicer 1 from different species. To design antisense morpholino oligonucleotides (Mos) we first defined the regions of the $5^{\prime}$ end of Xdicer 1 transcripts showing the highest conservation among different alleles and polymorphic forms. To this aim, we amplified by RT-PCR and sequenced the 112 bp region upstream of the first ATG from different batches of embryos at various stages. This analysis identified two forms of Xdicer1 transcripts and revealed conserved regions, which were used as target for the two Mos used in this work.

The specificity and efficiency of the two Mos, Xdcr-Mo1 and Xdcr-Mo2, was confirmed by microinjecting reporter constructs carrying a normal or mutated Mo target sequence (Fig. 1 and results not shown), and by comparing the expression of retinal miRNAs between normal and Mo-injected embryos (Supplementary Fig. 1).

We focused on the effects generated by dicer knockdown in Xenopus retinal development. Embryos were injected with $10 \mathrm{nl}$ of 125 microM Xdcr-Mo1 or Xdcr-Mo2 in one dorsal cell at 4 cell-

Abbreviations used in this paper: BrdU, bromodeoxyuridine; GFP, green fluorescent protein; Mo, morpholinooligonucleotide; Morphant, Mo-injected embryo; St., developmental stage; Stdev, standard deviation; S.e.m., standard error of the means.

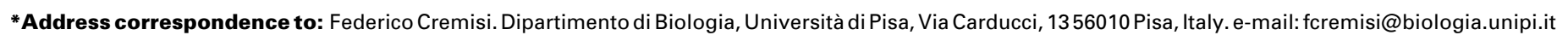

Electronic Supplementary Material for this article, consisting of 5 additional figures, is available online at: http://dx.doi.org/10.1387/ijdb.082646sd 
stage. Embryos injected with the same amount of control-Mo were indistinguishable from wild type (wt) embryos (not shown). Embryos injected with Xdcr-Mos were traced and selected by GFP mRNA coinjection. Microinjection of each Xdcr-Mo at 4-cell stage produced the same effects. The eyes of Mo-injected embryos (morphants) at the stage of swimming tadpole (st. 42) were

A

\begin{tabular}{|c|c|c|}
\hline XdcrMo2 & \multicolumn{2}{|c|}{\begin{tabular}{|l|l} 
& XdcrMo1 \\
\end{tabular}} \\
\hline \multicolumn{3}{|c|}{ 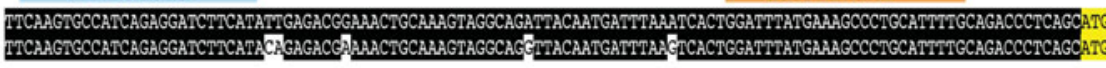 } \\
\hline \multicolumn{3}{|l|}{ WT-Mo2-target } \\
\hline CMVpromoter & CAAGTGCCATCAGAGGATCTTCATA & GFP \\
\hline \multicolumn{3}{|l|}{ MUT-Mo2-target } \\
\hline CMVpromoter & CA $g$ GTGCC TCAGA GATCT:CA:A & GFP \\
\hline
\end{tabular}

WT-Mo1-target

CMVpromoter GGATTTATGAAAGCCCTGCATTTTG GFP

MUT-Mo1-target

CMVpromoter GcATaTATGAAgGCCCTgCATTTcG GFP

B

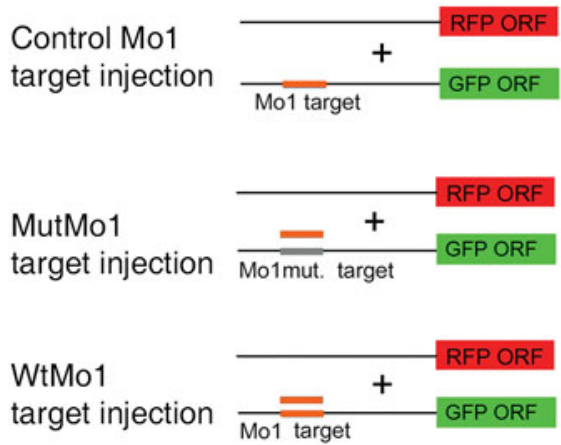

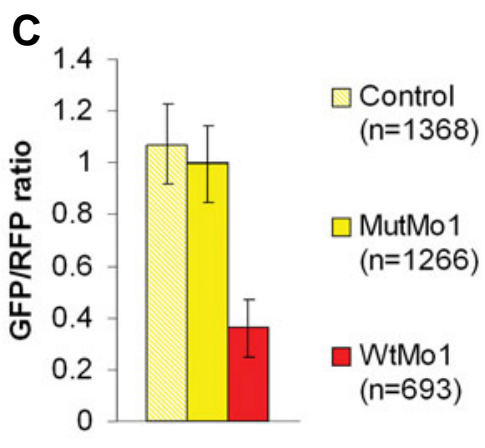
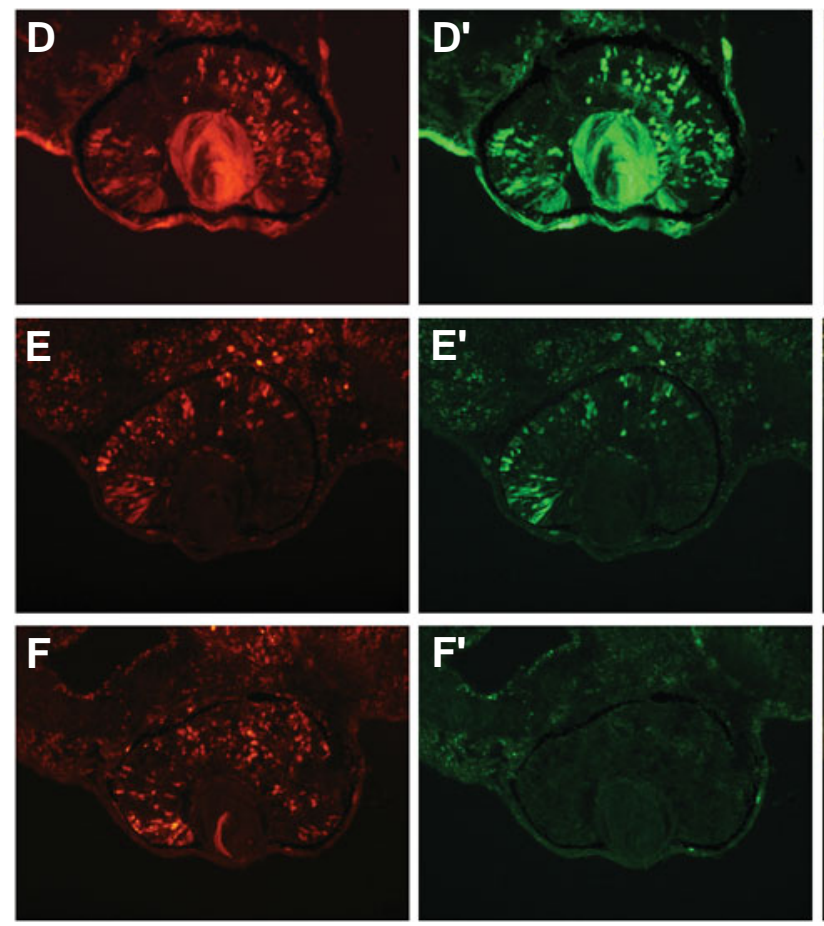

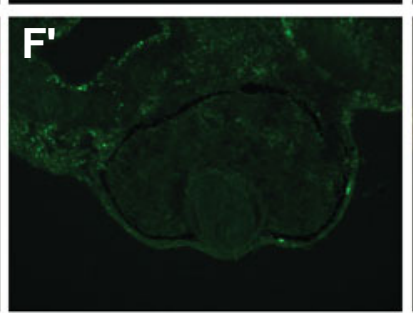

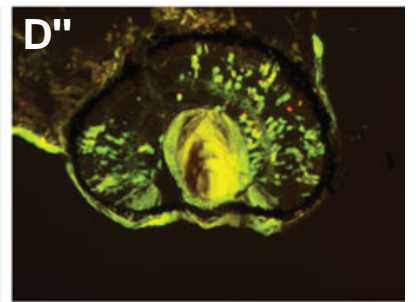
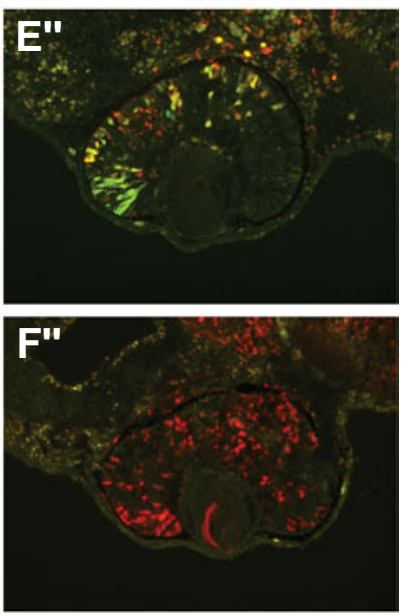

Fig. 1. In vivo GFP reporter activity of XdcrMo-target constructs. Sequence alignment in the top panel (A) shows the two forms of Xdicer1 obtained by the analysis, with mismatches indicated in white boxes. The target sequences of Xdcr-Mo1 and Xdcr-Mo2, which are the two Mos chosen for functional analysis, are shown in light blue and orange colours, respectively. Constructs carrying the GFP reporter activity under the control of normal (WT) or mutated (MUT) Xdcr-Mo target sequences were cloned in pCS2 vector under CMV transcriptional control, as shown in (A). In the MUT constructs, the small letters indicate inserted mutations. (B) Scheme of the strategy followed to assay Xdcr-Mo1 ability to specifically inhibit its target. Red Fluorescent Protein (RFP) construct was always co-injected into early embryo as an internal standard, together with WT or MUT constructs, without (Control Mo1 target injection) or with (MutMo1; Mo1 target injection) Xdcr-Mo1. (C) The ratio between GFP and RFP positive cells ( $n=$ number of cells analysed in three independent experiments) of mature embryonic retinas at st. 42. Bars indicate standard error of the mean. Notably, only WtMo1 target injection (WtMo1) significantly decreases the ratio $(p<0.001)$, indicating the specificity of Xdcr-Mo1 to inhibit its specific target. Comparable results were obtained when injecting WtMo2 or MutMo2 constructs, with or without Xdcr-Mo2 (not shown). (D-F") Examples of retinas after Control Mo1 target injection without any Mo (D-D"), MutMo1 target injection with Xdcr-Mo1 $\left(E-E^{\prime \prime}\right)$, or Mo1 Target injection with Xdcr-Mo1 $\left(F-F^{\prime \prime}\right)$. (D, E, F) RFP detection; $\left(D^{\prime}, E^{\prime}, F^{\prime}\right)$ GFP detection; $\left(D^{\prime \prime}, E^{\prime \prime}, F^{\prime \prime}\right)$ merge. 

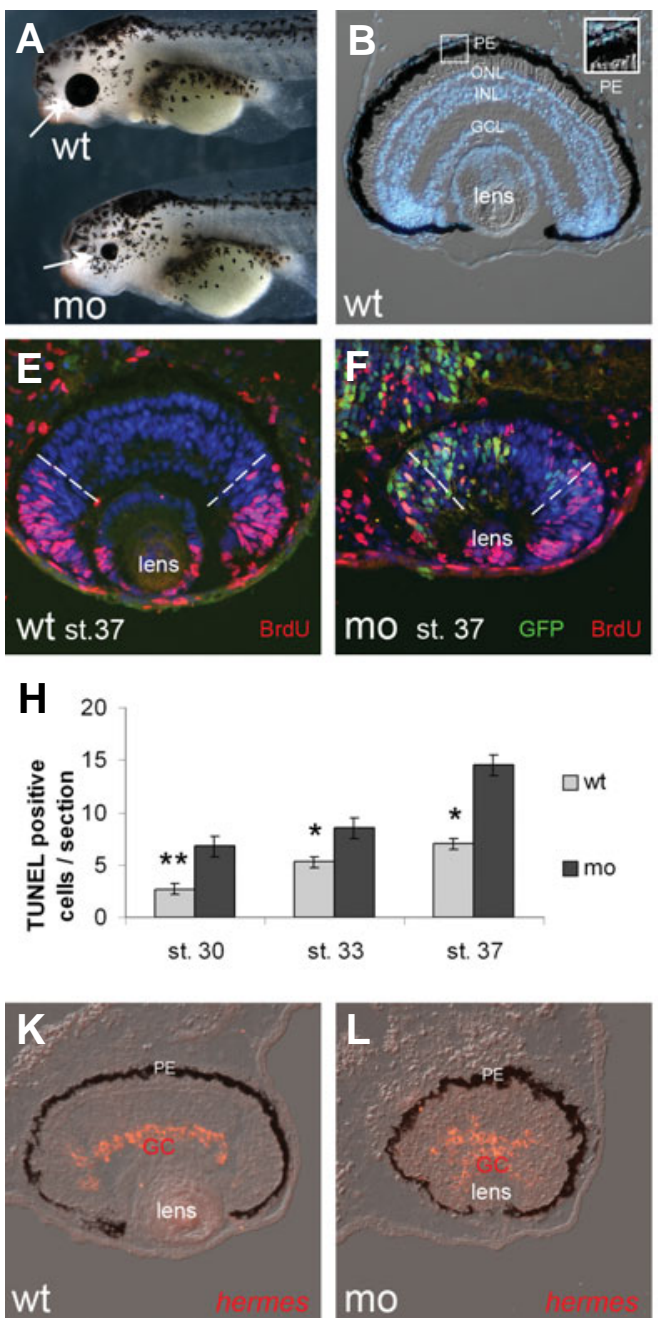
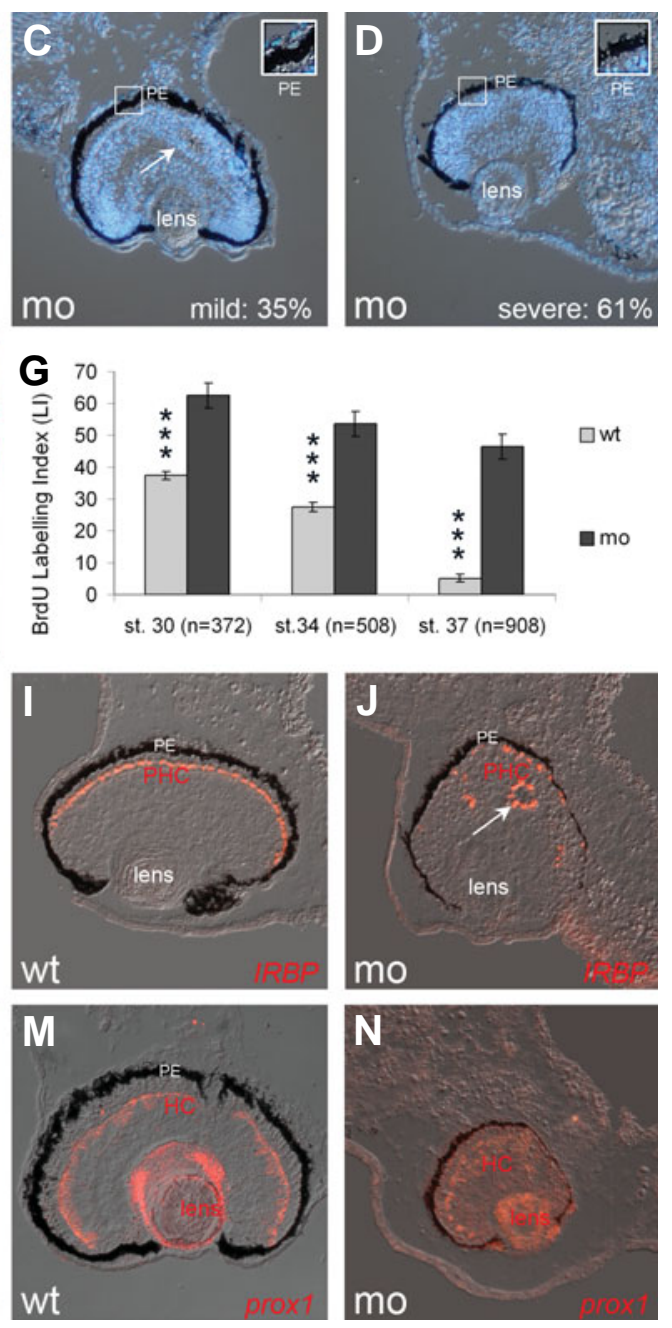

Fig. 2. Dicer down-regulation affects retinal cell lamination, delays the exit from the cell cycle and promotes cell death. (A) Comparison between morphant (mo) and wild type (wt) eyes (arrows) at st. 42. Embryos were injected with $10 \mathrm{nl}$ of $125 \mathrm{nM}$ Xdcr-Mo1 in one dorsal cell at 4 cellstage embryos. Injected embryos were traced and selected by GFP mRNA coinjection (300 ng, not shown). (B-D) Nuclear Hoechst staining of wt (B) and morphant $(C, D)$ eye sections. PE: pigmented epithelium, ONL: outernuclear layer, INL: inner nucler layer, GCL: ganglion cell layer. Although lens and PE morphology does not appear to be affected in morphants, the thickness of morphant PE is clearly reduced compared to control, as shown in box in (B) (control) and $(C, D)$ (mild and severe morphant phenotypes, respectively). (E-G) BrdU labelling index (LI), obtained by the analysis of wt and mo embryos after $8 \mathrm{~h} \mathrm{BrdU}$ incorporation. (G) Statistical analysis of BrdU-positive cells (red in E, F, and Supplementary Fig. 3) in the central aspect of retinal sections (delimited by dashed lines in E, F). GFP traces Xdcr-Mo1 injected cells. A significant $L I$ increase (triple asterisk: $p$ $<0.001$, student ttest) was observed in morphants compared to wt. Error bars show s.e.m., n: number of cells. (H) Statistical analysis of apoptotic TUNEL-positive cells (Supplementary Fig. 4) in mo and wt retinas ( $n=69$ and 482, respectively) at different stages. Mo showed a significantly higher number of apoptotic cells/section than wt (double asterisk: $p<0.01$, single asterisk $p<0.05$ ). Error bars: s.e.m. (I-N) In situ hybridisation of Xenopus celltype specific markers (Decembrini et al., 2006) on st. 42 mo and wt retinal sections: Xirbp (photoreceptors, PHC), Xhermes (ganglion cells, GC), Xprox1 (horizontal cells, HC). Although expressing the specific markers, retinal cells are not properly layered in mo (J,L,M) compared to wt (I,L,N).

inactivation might account for the decreased eye size, as it occurs for limb size in mouse (Harfe et al., 2005). According to previous studies (Lunardi et al., 2006), control-Mo injection did not affect cell proliferation and cell death (not shown).

Even if dicer knockdown dramatically affects retinal development and differentiation, it does not prevent the expression of a number of retinal cell markers such as Xirbp, Xhermes, Xprox1 (Fig. $2 \mathrm{I}-\mathrm{N}$ ), Xotx5b (Fig. $3 \mathrm{C}, \mathrm{D}$ ) and Xotx2 (Fig. $3 \mathrm{G}, \mathrm{H}$ ). This observation suggests that dicer downregulation is somehow compatible with the capability of retinal progenitor cells to progress toward neural differentiation. Thus, an interesting question is whether in vertebrates, as in C. elegans (Moss et al.,1997; Slack et al., 2000), miRNAs may affect the developmental timing. We compared the time of translation of Xotx5b and Xotx2, two key factors controlling the timing of retinal histogenesis, between morphants and control embryos. Whereas Xotx5b and Xotx2 mRNAs were present from early developmental stages both in morphants and in controls (st. 33, Supplementary Fig. 4;Viczian et al., 2003), the onset of both Xotx5b and Xotx2 proteins detection was delayed in morphants compared to controls. In partly injected embryos, we compared non-injected (GFP-negative) aspects of retinas to Mo-injected (GFP-positive) regions within the same eye. Non-injected (GFP-negative) aspects of retinas showed normal layering (Fig. $3 \mathrm{~A}-\mathrm{F}$ ). In these parts of the retinas, Xotx5b and Xotx2 expression is comparable to that of wt retinas (Decembrini et al., 2006). Conversely, neither Xotx5b nor Xotx2 are detectable in GFP-positive cells at st. 37 (Fig. 3 A,B) and 42 (Fig. $3 \mathrm{E}, \mathrm{F}$ ), respectively. Protein detection is delayed in Mo-injected cells to later stages, namely st. 42 (Xotx5b, Fig. 3 C,D) and st. 45 (Xotx2, Fig. 3 G,H). Embryos injected with control morpholino showed no such delayed expression of the two proteins (Supplementary Fig. 5).

\section{Discussion}

By dicer inactivation in Xenopus retinal progenitor cells, we found that miRNAs play a crucial role in cell cycle control and survival and are necessary to set the correct timing of translation of key genes of retinal histogenesis. Although miRNAs are expected to directly inhibit protein translation, our results indicate a 


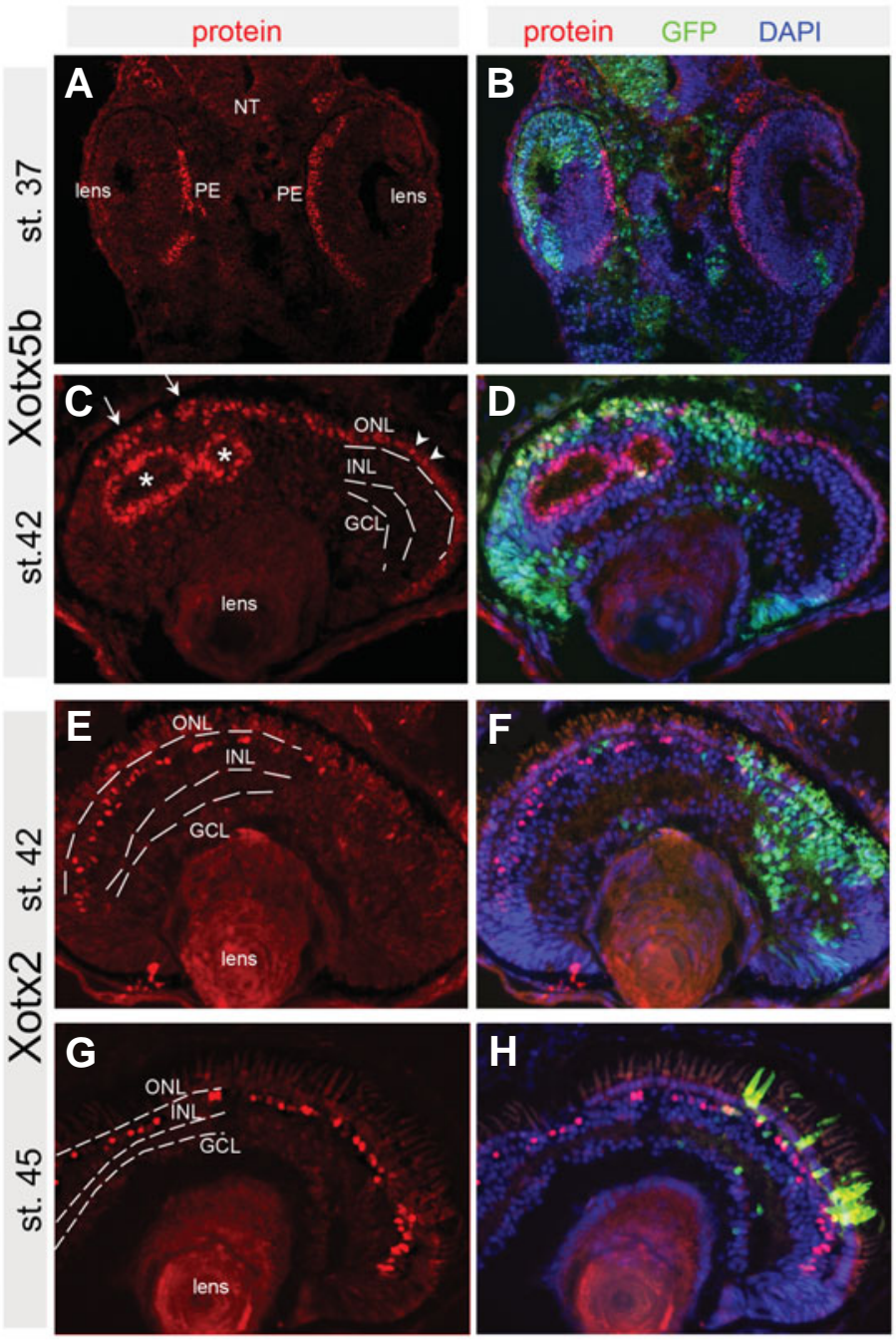

Fig. 3. Dicer down-regulation delays the translation of Xotx5b and Xotx2. (A-H) Typical immunodetections (red staining) of Xotx5b (A-D) and Xotx2 (E-H) on retinal sections of morphants at different developmental stages, obtained in three different experiments ( $n>15$ embryos in each experiment). GFP (green) traces injected cells in $(B, D, F, H)$; DAPI (blue) stains nuclei in $(B, D, F, H)$. NT: neural tube; $P E$ pigmented epithelium; ONL: outer nuclear layer, INL: inner nuclear layer, GCL: ganglion cell layer. The non-injected (GFP-negative) aspects of mo retinas showed normal layering, which is highlighted by dashed lines. In these parts of the retinas, Xot $\times 5 b$ and Xot $x 2$ expression is comparable to that of wt retinas (Decembrini et al., 2006). Conversely, Xotx $5 \mathrm{~b}$ and Xotx2 are not detectable in GFP-positive cells at st. $37(A, B)$ and $42(E, F)$, respectively. Protein detection is delayed in Mo-injected cells to later stages, namely st. 42 (Xotx5b, C,D) and st. 45 (Xotx2, G, H). Asterisk in (C) shows rosette-like structures. In (H), the Mo-injected aspect of the retina, which is GFPlabelled, shows Xotx2-positive nuclei (labelled in red). In (C), arrowheads indicate a typical monolayer of Otx5b-positive, normal photoreceptor nuclei, whereas arrows point at multilayered GFP/Xotx $5 b$ double-positive nuclei.

facilitating rather than an inhibitory role of miRNAs on the translation of the key cell fate factors Xotx5b and Xotx2. This is reminiscent of the mode of action of the let-7 (Slack et al., 2009) and lin4 (Moss et al., 1997) miRNAs, which generate heterochronic phenotypes in $C$. elegans mutants. Indeed, these two miRNAs indirectly facilitate the translation of developmental genes, by repressing the translation of inhibitory RNA binding proteins. We speculate that such kind of miRNAs could exist also in vertebrates and that in Xenopus they could indirectly support the translation of Xotx $5 \mathrm{~b}$ and Xotx2, and therefore the generation of photoreceptors and bipolar cells. Indeed, in a preliminary study, we for instance observed that the vertebrate counterpart of lin-4 (miR-125b) is expressed in the embryonic retina and that its inactivation by antisense oligonucleotide lipofection dramatically reduces the proportion of bipolar neurons in lipofected retinas. However, the overexpression of this miRNA alone is not sufficient to rescue the effects of dicer knockdown (unpublished), suggesting that a complex miRNA regulatory network may in fact exist for retinogenesis.

A crucial link between cell cycle and cell fate has been shown in Xenopusretinal progenitor cells (Moore etal., 2002; Ohnuma etal., 2002; Casarosa et al., 2003; Cremisi et al., 2003). In fact, there is a close relation between the time a retinal progenitor cell exits from the cell cycle (cell birth date) and its differentiation fate. As a consequence, anticipating or delaying the cell birth date of retinal progenitors affects the type of neurons they generate. Cdk2/ cyclinA2 overexpression delays retinal cell birth date and supports the expression of Xotx2 protein, thus causing an increase of the proportion of bipolar neurons (Casarosa et al., 2003; Decembrini et al., 2006). Similarly to cdk2/cyclinA2 overexpression, dicer morpholino injection also delays the average retinal cell birth date (Fig. 2G, Supplementary Fig. 2). However, dicer downregulation does not support Xotx 2 expression, rather it delays it, as it occurs for Xotx5b (see Fig. 3).

Thus, the effects of dicer knockdown can not be simply explained as a consequence of delayed cell birth date.

Although delayed, the translation of Xotx5b and Xotx2 in morphants is not repressed and, at a qualitative analysis, the proportion of cells expressing the two proteins eventually appears to be comparable in morphants and controls. This favours the hypothesis that both photoreceptors and bipolar cells may form properly in morphants, even if later compared to controls. Normal photoreceptors and bipolar cells segregate in two distinct cell layers in a timely ordered process and in coordination with the other retinal cell types. Thus, the heterochronic generation of these two cell types is compatible with a general disorganisation of layers, including the formation of rosette-like structures.

In conclusion, dicer inactivation generates a retinal heterochronic phenotype, namely delay of both retinal cell birth-date and translation of key genes required for the generation of late retinal cell types. This supports the idea that specific miRNAs are part of a cell clock machinery timing retinal neurogenesis.

\section{Materials and Methods}

\section{Design and use of morpholino oligonucleotides}

RT-PCR of Xenopus laevis dicer was carried out using forward 5 'atgcatttcaagtgccatca and reverse 5 'aaaagggacccattggagag primers. Mos, including a standard control morpholino oligonucleotide (control morpholino), were designed and supplied by Gene-Tools. A master mix of 30 $\mathrm{pg} / \mathrm{nl}$ each of pCs2-RFP (a kind gift of Dr. Marina Mione) and either Wt-Motarget or Mut-Mo-target GFP reporter constructs was previously prepared. Either Xdcr-Mo1, Xdcr-Mo2 or control Mo was added at the final concentration of 125 microM, when indicated, and $10 \mathrm{nl}$ of the mix were injected at 4cell stage into one dorsal blastomere. The analysis of RFP and GFP positive cells was carried out at st. 42 on 12 micron cryosections, under 
normal epifluorescence.

\section{Microarray screening}

miRNAs expressed in the developing Xenopus retina were selected by probing an Exiqon miRCURY'TM LNA Array version 7.1 with 2 micrograms of total RNA extracted from a pool of st.33 dissected retinas $(n=40)$, following the manufacturer method. The capture probes showing highest signals were chosen for further validation by ISH.

\section{Morphological analysis, immunodetection and ISH}

$\mathrm{PE}$ analysis was performed on microphotographs of either control or dcr-Mo injected embryos (including both mild and severe phenotype), using the ImageJ software. The thickness of three different pictures of each retina, for a total number of 14 retinas, was measured by the measure tool and the average $P E$ thickness of morphants was calculated as percentage of the control average thickness.

The whole mount protocol of ISH of miRNAs (Wienholds et al., 2005; Kloosterman et al., 2006) is a slight modification of the common ISH protocol. The significant difference is the probe nature and the temperature of hybridisation and of the washes, which is usually set $20-22^{\circ} \mathrm{C}$ lower than the Tm of the LNA modified oligonucleotide used as probe. We used 15-22 nt long, Dig-pre-labelled LNATM probes (Exiqon), complementary to the mature sequence of miRNAs. Detection was carried out at RT by BMP purple staining for 24-36 hrs. Negative control probes did not significantly stain (not shown). List of probes used:

mir-298: 5' GGAAGAACAGCCCTCCTCTGCC

mir-375: 5' TCACGCGAGCCGAACGAACAAA

mir-452: 5' GTCTCAGTTTCCTCTGCAAACA

mir-381: 5' ACAGAGAGCTTGCCCTTGTATA

mir-125a: 5' CACAGGTTAAAGGGTCTCAGGGA

mir-347: 5' TGGGCGACCCAGAGGGACA.

mRNA ISH and immunodetection were performed as previously described (Casarosa et al., 2003; Decembrini et al., 2006).

\section{BrdU labelling index}

Embryos were co-injected at 4-cell stage in a dorsal blastomere with Xdcr-Mo1 and GFP as tracer. Embryos were then injected with BrdU (100 $\mathrm{nl}$ of $15 \mathrm{mg} / \mathrm{ml} \mathrm{BrdU}$ solution) at the stages indicated, fixed after $8 \mathrm{hrs}$ and eventually processed for further analysis as described (Decembrini et al., 2006).

\section{TUNEL assay}

TUNEL assay was performed by an ApopTag Peroxidase Kit (Serologicals) on 12 micron cryostat sections, following the manufacturer's instruction.

\section{Aknowledgements}

We thank Dario Bressan for technical assistance and Robert Vignali, Giuseppe Rainaldi and Letizia Pitto for helpful discussion. This work was supported by Telethon grant no. GGP07275, by Italian Department for Foreign Affairs (MAE) and the Italian Department of University and Scientific Research (MIUR).

\section{References}

AMBROS, V. (2000). Control Of Developmental Timing In Caenorhabditis Elegans. Curr Opin Genet Dev 10: 428-433.
BARTEL, DP. (2004). Micrornas: Genomics, Biogenesis, Mechanism, And Function. Ce//116: 281-297.

BERNSTEIN, E., KIM, SY., CARMELL, MA., MURCHISON, EP., ALCORN, H., LI, MZ., MILLS, AA., ELLEDGE, SJ., ANDERSON, KV. and HANNON, GJ. (2003). Dicer Is Essential For Mouse Development. Nat Genet 35: 215-217.

CASAROSA, S., AMATO, MA., ANDREAZZOLI, M., GESTRI, G., BARSACCHI, G. and CREMISI, F. (2003). Xrx1 Controls Proliferation And Multipotency Of Retinal Progenitors. Mol Cell Neurosci22: 25-36.

CREMISI, F., PHILPOTT, A. and OHNUMA, S. (2003). Cell Cycle And Cell Fate Interactions In Neural Development. Curr Opin Neurobio/13: 26-33.

DECEMBRINI, S., ANDREAZZOLI, M., VIGNALI, R., BARSACCHI, G. and CREMISI, F. (2006). Timing The Generation Of Distinct Retinal Cells By Homeobox Proteins. PLOS Bio/4: e272.

GIRALDEZ, AJ., CINALLI, RM., GLASNER, ME., ENRIGHT, AJ., THOMSON, JM., BASKERVILLE, S., HAMMOND, SM., BARTEL, DP. and SCHIER, AF. (2005). Micrornas Regulate Brain Morphogenesis In Zebrafish. Science 308: 833-838.

HARFE, BD., MCMANUS, MT., MANSFIELD, JH., HORNSTEIN, E. and TABIN, CJ. (2005). The Rnaseiii Enzyme Dicer Is Required For Morphogenesis But Not Patterning Of The Vertebrate Limb. Proc Nat/Acad SciUS A 102: 10898-10903.

HATAKEYAMA, J., TOMITA, K., INOUE, T. and KAGEYAMA, R. (2001). Roles Of Homeobox And Bhlh Genes In Specification Of A Retinal Cell Type. Development 128: 1313-1322.

KLOOSTERMAN, WP., WIENHOLDS, E., DE BRUIJN, E., KAUPPINEN, S. and PLASTERK, RHA. (2006). In Situ Detection Of Mirnas In Animal Embryos Using Lna-Modified Oligonucleotide Probes. Nat Methods 3: 27-29.

LIVESEY, FJ. and CEPKO, CL. (2001). Vertebrate Neural Cell-Fate Determination: Lessons From The Retina. Nat Rev Neurosci2: 109-118.

LUNARDI, A., CREMISI, F. and DENTE, L. (2006). Dystroglycan Is Required For Proper Retinal Layering. Dev Bio/290: 411-420.

MOORE, KB., SCHNEIDER, ML. and VETTER, ML. (2002). Posttranslational Mechanisms Control The Timing Of Bhlh Function And Regulate Retinal Cell Fate. Neuron 34: 183-195.

MOSS, EG., LEE, RC. and AMBROS, V. (1997). The Cold Shock Domain Protein Lin28 Controls Developmental Timing In C. Elegans And Is Regulated By The Lin-4 Rna. Cel/88: 637-646.

NAKAGAWA, S., TAKADA, S., TAKADA, R. and TAKEICHI, M. (2003). Identification Of The Laminar-Inducing Factor: Wnt-Signal From The Anterior Rim Induces Correct Laminar Formation Of The Neural Retina In Vitro. Dev Bio/260: 414-425.

OHNUMA, S., HOPPER, S., WANG, KC., PHILPOTT, A. and HARRIS, WA. (2002). Co-Ordinating Retinal Histogenesis: Early Cell Cycle Exit Enhances Early Cell Fate Determination In The Xenopus Retina. Development 129: 2435-2446.

SLACK, FJ., BASSON, M., LIU, Z., AMBROS, V., HORVITZ, HR. and RUVKUN, G. (2000). The Lin-41 Rbcc Gene Acts In The C. Elegans Heterochronic Pathway Between The Let-7 Regulatory Rna And The Lin-29 Transcription Factor. Mo/ Cell 5: 659-669.

VICZIAN, AS., VIGNALI, R., ZUBER, ME., BARSACCHI, G. and HARRIS, WA. (2003). Xotx5B And Xotx2 Regulate Photoreceptor And Bipolar Fates In The Xenopus Retina. Development 130: 1281-1294.

WIENHOLDS, E., KLOOSTERMAN, WP., MISKA, E., ALVAREZ-SAAVEDRA, E., BEREZIKOV, E., DEBRUIJN, E., HORVITZ, HR., KAUPPINEN, S. and PLASTERK, $\mathrm{RH}$. (2005). Microrna Expression In Zebrafish Embryonic Development. Science 309: 310-311. 


\section{Related, previously published Int. J. Dev. Biol. articles}

See our recent Special Issue Developmental Biology in Poland edited by Tarkowski, Maleszewski and Kloc at: http://www.ijdb.ehu.es/web/contents. php?vol=52\&issue=2-3

See our recent Special Issue Ear Development edited by Fernando Giraldez and Bernd Fritzsch at: http://www.ijdb.ehu.es/web/contents.php?vol=51\&issue=6-7

Cloning and developmental expression of the soxB2 genes, sox14 and sox21, during Xenopus laevis embryogenesis Doreen D. Cunningham, Zhuo Meng, Bernd Fritzsch and Elena Silva Casey Int. J. Dev. Biol. (2008) 52: 2586-2586

Early neural cell death: numbers and cues from the developing neuroretina Ana I. Valenciano, Patricia Boya and Enrique J. de la Rosa Int. J. Dev. Biol. (2008) 52: 2446-2446

\section{Spatiotemporal clustering of cell death in the avian forebrain proliferative zone} Christine J. Charvet and Georg F. Striedter Int. J. Dev. Biol. (2008) 52: 345-352

A network of growth and transcription factors controls neuronal differentation and survival in the developing ear Hortensia Sánchez-Calderón, Marta Milo, Yolanda León and Isabel Varela-Nieto Int. J. Dev. Biol. (2007) 51: 557-570

Expression and regulation of Xenopus CRMP-4 in the developing nervous system Jacob Souopgui, Tiemo J. Klisch, Tomas Pieler and Kristine A. Henningfeld Int. J. Dev. Biol. (2007) 51: 339-343

$\mathrm{XSu}(\mathrm{H}) 2$ is an essential factor for gene expression and morphogenesis of the Xenopus gastrula embryo Motoaki Ito, Tomohisa Katada, Seiji Miyatani and Tsutomu Kinoshita Int. J. Dev. Biol. (2007) 51:27-36

Retinal stem cells and regeneration Ala Moshiri, Jennie Close and Thomas A. Reh Int. J. Dev. Biol. (2004) 48: 1003-1014

Retinal stem cells in vertebrates: parallels and divergences Marcos A. Amato, Emilie Arnault and Muriel Perron Int. J. Dev. Biol. (2004) 48: 993-1001

Cell death in the developing vertebrate retina Elena Vecino, María Hernández and Mónica García Int. J. Dev. Biol. (2004) 48: 965-974

XMam1, the Xenopus homologue of mastermind, is essential to primary neurogenesis in Xenopus laevis embryos.

Tomohisa Katada and Tsutomu Kinoshita Int. J. Dev. Biol. (2003) 47: 397-404

Notch activity is required to maintain floorplate identity and to control neurogenesis in the chick hindbrain and spinal cord. Isabelle le Roux, Julian Lewis and David Ish-Horowicz Int. J. Dev. Biol. (2003) 47: 263-272

Cysteine-rich region of X-Serrate-1 is required for activation of Notch signaling in Xenopus primary neurogenesis.

Tomomi Kiyota and Tsutomu Kinoshita Int. J. Dev. Biol. (2002) 46: 1057-1060

Early neurogenesis in Amniote vertebrates. N M Le Douarin Int. J. Dev. Biol. (2001) 45: 373-378

2006 ISI **Impact Factor $=3.577^{\star *}$

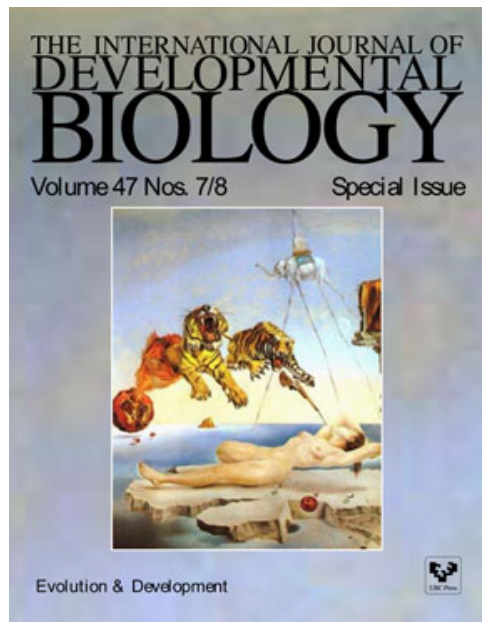

